\title{
Protection of insulin-like growth factor 1 on experimental peripheral neuropathy in diabetic mice
}

\author{
HUA WANG, HAO ZHANG, FUMING CAO, JIAPING LU, JIN TANG, \\ HUIZHI LI, YIYUN ZHANG, BO FENG and ZHAOSHENG TANG
}

\begin{abstract}
Department of Endocrinology, Shanghai East Hospital, Tongji University School of Medicine, Shanghai 200120, P.R. China
\end{abstract}
Received December 24, 2017; Accepted July 19, 2018

DOI: $10.3892 / \mathrm{mmr} .2018 .9435$

\begin{abstract}
The present study investigated whether insulin-like growth factor-1 (IGF-1) exerts a protective effect against neuropathy in diabetic mice and its potential underlying mechanisms. Mice were divided into four groups: $\mathrm{Db} / \mathrm{m}$ (control), db/db (diabetes), IGF-1-treated $\mathrm{db} / \mathrm{db}$ and IGF-1-picropodophyllin (PPP)-treated db/db. Behavioral studies were conducted using the hot plate and von Frey methods at 6 weeks of age prior to treatment. The motor nerve conduction velocity (NCV) of the sciatic nerve was measured using a neurophysiological method at 8 weeks of age. The alterations in the expression levels of IGF-1 receptor (IGF-1R), c-Jun N-terminal kinase (JNK), extracellular signal-regulated kinase (ERK), p38 and effect of IGF-1 on the sciatic nerve morphology were observed by western blotting and electron microscopy. Compared with the control group, the diabetes group developed hypoalgesia after 12 weeks, and neurological lesions improved following an intraperitoneal injection of recombinant (r)IGF-1. The sciatic NCV in the diabetes group was significantly lower compared with the control group. The sciatic NCV improved following rIGF-1 intervention; however, was impaired following administration of the IGF-1 receptor antagonist, PPP. The myelin sheath in the sciatic nerve of the diabetes group was significantly more impaired compared with the control group. The myelin sheath in the sciatic nerves of the rIGF-1-treated group was significantly improved compared with the diabetes group; whereas, they were significantly impaired following administration of the IGF-1R inhibitor. In addition, the expression of IGF-1R, phosphorylated (p)-JNK and p-ERK of sciatic nerves in the $\mathrm{db} / \mathrm{db}$ mice was significantly increased following treatment with IGF-1. The expression levels of these proteins were significantly lower in
\end{abstract}

Correspondence to: $\mathrm{Dr}$ Zhaosheng Tang, Department of Endocrinology, Shanghai East Hospital, Tongji University School of Medicine, 150 Jimo Road, Shanghai 200120, P.R. China

E-mail: zhaoshengtang@aliyun.com

Key words: insulin-like growth factor-1, diabetes mellitus, diabetic peripheral neuropathy, insulin-like growth factor-1 receptor, sciatic nerve the IGF-1-PPP group compared with the IGF-1 group; however, no significant difference was observed in the expression levels of p-p38 following treatment with IGF-1. The results of the present study demonstrated that IGF-1 may improve neuropathy in diabetic mice. This IGF-1-induced neurotrophic effect may be associated with the increased phosphorylation levels of JNK and ERK, not p38; however, it was attenuated by administration of an IGF-1R antagonist.

\section{Introduction}

An epidemiological survey demonstrated that the prevalence of diabetes in adults $\geq 18$ years old in China was $\leq 11.6 \%$ (1). Diabetic neuropathy is one of the three principal complications of diabetes, occurring earlier and more frequently compared with other common complications, including nephropathy and retinopathy (2). Overall, $\geq 50 \%$ of patients with diabetes exhibit a certain degree of diabetic peripheral neuropathy (DPN); the prevalence of DPN has been reported to be $16.2-28.9 \%$ (3). Patients with diabetes and DPN may exhibit alterations in sensation, movement or organ function, depending on the type of nerves involved (4). Sensory neuropathy may cause numbness to touch and vibration, reduced position sense associated with poorer coordination and balance, and reduced sensitivity to pain and alterations in temperature $(5,6)$. Motor neuropathy may cause impaired balance and coordination, and most commonly, muscle weakness $(5,6)$. The detailed pathogenesis of peripheral nervous system dysfunction associated with diabetes is unknown. Previous studies have demonstrated that this pathogenesis may be associated with long-term hyperglycemia, increased production of glycosylation end products, alterations in microcirculation and neurotrophic factors, oxidative stress and autoimmune disorders $(7,8)$. In addition, it has been reported that the polyol pathway is the principal source of diabetes-induced oxidative stress in nerves (9); however, there are no clinically effective treatments for DPN. Insulin therapy may regulate blood glucose; however, a number of patients with diabetes still develop DPN. Therefore, understanding the pathogenesis of neuropathy in patients with diabetes is necessary for the development of effective treatments. Recently, the roles of insulin-like growth factor 1 (IGF-1) in the prevention/treatment of diabetes and associated complications have become a subject of interest. 
IGF-1 is a single-stranded polypeptide of 70 amino acids and has been considered to be a multipotent neurotrophic factor that is widely expressed in the body (10). A previous study has demonstrated that IGF-1 ameliorated increased oxidative stress in the liver, suggesting that IGF-1 may regulate mitochondrial function and oxidative stress (11). In addition, IGF-1 was observed to improve mitochondrial function in vitro and in vivo (12). IGF-1 was reported to provide trophic support for numerous neurons of the peripheral and central nervous systems (13); DPN has been proposed to be closely associated with IGF-1 (14). A recent study suggested that IGF-1 serves a role for neurotrophic function in the treatment of diabetic neuropathy (15); however, the molecular and cellular mechanisms underlying the effects of IGF-1 on DPN require further investigation.

The present study aimed to determine the neurotrophic effects of IGF-1 in a mouse model of diabetic neuropathy. The potential underlying mechanisms were examined using a mouse model of neuropathy in $\mathrm{db} / \mathrm{db}$ mice. Additionally, the present study investigated the peripheral drug-specific target of IGF-1. The results of the present study may aid the development of novel clinical therapeutic strategies and provide a basis for future investigations into the pathogenesis of DPN.

\section{Materials and methods}

Ethical approval. All experimental procedures were approved by the Ethics of Animal Experiments Committee of the Tongji University School of Medicine (Shanghai, China). The present study was conducted according to the Institutional Animal Care and Research Advisory Committee of the Medical Department of Tongji University.

Blood chemistry. The onset of diabetes was confirmed by measuring fasting blood glucose levels. One drop of tail blood was analyzed using a standard glucometer (Contour TS meter, Bayer AG, Leverkusen, Germany) beginning from 8 weeks of age to 20 weeks of age and repeated every 2 weeks to document the progression of diabetes.

Animals. In total, 45 male C57 BKS db/db mice (cat. no. N000180) weighing 50-60 g were purchased from Nanjing Biomedical Research Institute of Nanjing University (Nanjing, China). In total, 15 male C57 BKS db/m mice (cat. no. 201500531244) weighing 25-30 g were purchased from Shanghai Laboratory Animal Co., Ltd. (Shanghai, China). The homozygous $(\mathrm{db} / \mathrm{db})$ mice were used as a model of type 2 diabetes; whereas, heterozygous mice $(\mathrm{db} / \mathrm{m})$ served as non-diabetic controls. Mice were housed in wire-bottomed cages in $22 \pm 1^{\circ} \mathrm{C}$ with a 12 -h light/dark cycle. The mice were raised on a commercial pellet diet (Jiangsu Province Collaborative Medicine Bioengineering Co., Ltd, Jiangsu, China) and were provided with access to tap water ad libitum. Mice were divided into four groups: Db/m group (control; $\mathrm{n}=15$ ), db/db group (diabetic model; $\mathrm{n}=15$ ), IGF-1-treated db/db mice (IGF-1 group; $n=15$ ) and the IGF-1-picropodophyllin (IGF-1-PPP)-treated db/db group $(n=15)$. The weight and blood glucose of each mouse were measured every 2 weeks from 8-20 weeks of age.
Animal treatments. Recombinant (r)IGF-1 was purchased from Sino Biological Inc. (Beijing, China), which was dissolved in normal saline and stored in aliquots at $-80^{\circ} \mathrm{C}$. PPP was purchased from Selleck Chemicals (Shanghai, China) and freshly dissolved in dimethyl sulfoxide at a concentration of $50 \mathrm{mM}$ prior to use. The IGF-1 group was intraperitoneally injected with rIGF-1 $(0.4 \mathrm{mg} / \mathrm{kg})$ every day. The IGF-1-PPP-treated $\mathrm{db} / \mathrm{db}$ group was intraperitoneally injected with PPP (20 mg/kg) and rIGF-1 ( $0.4 \mathrm{mg} / \mathrm{kg})$ every day from 8 weeks of age for $\sim 12$ weeks (16). Blood samples (0.8-1.2 $\mathrm{ml})$ were drawn from the abdominal aorta and anti-coagulated with $1 \mathrm{mg} / \mathrm{ml}$ EDTA. Subsequently, samples were used to determine serum levels of IGF-1 and IGF binding protein 3 (IGFBP-3). As described below, sciatic nerves were analyzed to determine motor nerve conduction velocity (MNCV) and morphology; the protein expression levels of IGF-1, IGF-1 receptor (IGF-1R), c-Jun N-terminal kinase (JNK), extracellular signal-regulated kinase (ERK) and p38 were additionally investigated.

\section{Behavioral studies}

Hot plate method. At 6 weeks of age, mice were placed on a glass plate within a transparent bottomless plastic chamber $(20 \times 10 \times 20 \mathrm{~cm})$ and were allowed to acclimate for $30 \mathrm{~min}$. Paw withdrawal latency (PWL) was considered as an index of the nociceptive threshold. At the beginning of the trials, the plantar surface of the mouse's paws were exposed to a light beam of radiant heat (cat. no. BME-410C; automatic thermal pain stimulator; Institute of Biomedical Engineering, Chinese Academy of Medical Sciences, Tianjin, China). Once the mouse lifted its paw, the light beam was turned off immediately; the duration between exposure to the light beam to the raising of the paw was recorded and denoted as the PWL. The hind paws were tested alternately at intervals of $5 \mathrm{~min}$. The cut-off time for heat stimuli was $30 \mathrm{sec}$.

Von Frey method. The Von Frey method was conducted as previously described (17). The 50\% paw withdrawal threshold (PWT) of mice was calculated as follows: A Plexiglas cubicle was placed on a nylon mesh and the mouse was placed in a bottomless glass box. The von Frey fibers (cat. no. NC12775-99; North Coast Medical, Inc., Morgan Hill, CA, USA) were used to stimulate the feet of the mouse's hind limbs for $<4 \mathrm{sec}$ and the mice remained in the Plexiglass box for $30 \mathrm{~min}$. Mice which appeared to lift or lick their feet were regarded as exhibiting a positive reaction, otherwise a negative score was reported.

MNCV measurements. Sciatic NCV was measured using a diabetic peripheral nerve injury-screening instrument (electromyography evoked potential meter; cat. no. NDI-097; Shanghai Haishen Medical Electronic Instrument Co., Ltd., Shanghai, China) as described in a previous study (18), with minor modifications at 8 weeks of age. Mice of the four groups were anesthetized in a prone position. The electrodes of the stimulus needle were positioned between the femur and the calcaneal tubercle; the electrode of the recording needle was placed via the site of the ankle of the sciatic nerve. The reference electrode was positioned between the stimulation and recording electrodes, the reference electrode was $1 \mathrm{~cm}$ 
from the recording electrode; all three types of electrodes were needle electrodes. A stimulus was applied with a single pulse square wave, $0.1 \mathrm{msec}$ wave width, 1.5-times the intensity of the threshold. Each of the two stimuli had an interval of $>6$ sec. The room temperature $\left(20.0 \pm 0.5^{\circ} \mathrm{C}\right)$ was strictly controlled and the animal body temperature was maintained at $37^{\circ} \mathrm{C}$. The distance between the stimulus electrode and the recording electrode was measured. The MNCV was calculated from the duration between the onset of the stimulation to the evoked potential of the muscle.

Tissue preparation and morphological observation. Mice were anesthetized with $40 \mathrm{mg} / \mathrm{kg}$ pentobarbital, the abdominal cavities of the mice were surgically opened following local disinfection. Blood samples $(0.8-1.2 \mathrm{ml})$ were obtained from the abdominal aorta, maintained at room temperature for 15 min prior to centrifugation in an Eppendorf tube containing EDTA (1 mg/ml) and subsequently centrifuged for $20 \mathrm{~min}$ at $2,000 \mathrm{x} \mathrm{g}$ at $4^{\circ} \mathrm{C}$. The supernatant was stored in an Eppendorf tube and preserved at $-70^{\circ} \mathrm{C}$ for further use. The mice were sacrificed via cervical dislocation. Subsequently, the sciatic nerve specimens were collected. The majority of the specimens were frozen in liquid nitrogen until further analysis, $2 \mathrm{~mm}$ long sections were fixed with $2.5 \%$ glutaraldehyde for $6 \mathrm{~h}$ at $4^{\circ} \mathrm{C}$. Subsequently, the samples were rinsed with $0.1 \mathrm{M}$ phosphate buffer and treated with $1 \% \mathrm{OsO}_{4}$ solution (Alfa Aesar; Thermo Fisher Scientific, Inc.) for $3 \mathrm{~h}$, dehydrated with a graded series of ethanol (30,50, 80 and 90\%) and propylene oxide, and embedded in epoxy resin (Shanghai Resin Factory Co., Ltd, Shanghai, China). Semi-thin sections (0.8-1 $\mu \mathrm{m})$ were stained with $1 \%$ toluidine blue (Sinopharm Chemical Reagent Co., Ltd., Shanghai, China) for histopathology at room temperature for $4 \mathrm{~min}$. Suitable areas of ultrathin $(80-90 \mathrm{~nm})$ nerve sections were selected for ultrastructural analysis. These were sectioned using a diamond knife (cat. no. 706602; Leica Microsystems GmbH, Wetzlar, Germany), mounted on a copper grid (Shanghai Huake Experimental Devices and Materials Co., Ltd., Shanghai, China), and stained with uranyl acetate for $30 \mathrm{~min}$ and lead citrate for $10 \mathrm{~min}$ at room temperature. Sections were analyzed and images were captured under a transmission electron microscope (Tecnai-12; FEI; Thermo Fisher Scientific, Inc.; magnification, x6,000; five fields of view analyzed).

ELISA analysis. The blood samples that were collected from the mice were analyzed. Blood samples from the abdominal aorta were centrifuged for $20 \mathrm{~min}$ at $2,000 \mathrm{x} \mathrm{g}$ at $4^{\circ} \mathrm{C}$. The serum was stored in an Eppendorf tube and preserved at $-70^{\circ} \mathrm{C}$ for further use. The serum IGF-1 and IGFBP-3 expression levels were measured using ELISA kits (cat. nos. DL-IGF1-Mu and DL-IGFBP3-Mu; Wuxi Donglin Sci \& Tech Development Co, Ltd., Nanjing, China), according to the manufacturer's protocol.

Western blot analysis. Sections of the sciatic nerve at 20 weeks of age and after 12 weeks of treatment from mice in each group were frozen in liquid nitrogen and preserved at $-80^{\circ} \mathrm{C}$ for further analysis. Samples were homogenized three times for $15 \mathrm{sec}$, in ice-cold radioimmunoprecipitation assay homogenization buffer (Merck KGaA, Darmstadt, Germany) containing Protease Inhibitor Cocktail Set III (cat. no. 539134; Thermo Fisher Scientific, Inc.) and Phosphatase Inhibitor Cocktail Set V (cat. no. 524629; Thermo Fisher Scientific, Inc.) using a PRO 200 homogenizer (PRO Scientific, Inc., Oxford, CT, USA). The homogenate was centrifuged at $4^{\circ} \mathrm{C}$ for $30 \mathrm{~min}$ at $12,000 \mathrm{x} \mathrm{g}$ and the supernatant was analyzed to detect the total protein concentration. The protein concentration was determined using a protein assay kit (DC Protein Assay kit; Bio-Rad Laboratories, Inc., Hercules, CA, USA). Samples $(40 \mu \mathrm{g})$ were separated via 10\% SDS-PAGE and transferred onto polyvinylidene difluoride membranes with an electrophoresis blotting transfer apparatus. The membranes were blocked with $5 \%$ milk powder for $1 \mathrm{~h}$ at $4^{\circ} \mathrm{C}$ and subsequently incubated with primary antibodies overnight at $4^{\circ} \mathrm{C}$. The following day, following three washes with Tris-buffered saline with $0.1 \%$ Tween-20, the membranes were incubated with the second antibody at room temperature for $1 \mathrm{~h}$. Bands were visualized following exposure to an enhanced chemiluminescence reagent (cat. no. 5978613; GE Healthcare Life Sciences, Shanghai, China) according to the manufacturer's protocol. Quantitative values were obtained from the densitometry of the western blotting bands using Gel-Pro Analyzer software (version 4.0; Media Cybernetics, Inc., Rockville, MD, USA). Antibodies against IGF-1 (1:100; cat. no. AF791-SP) and IGF-1R (1:100; cat. no. AF-305-SBD) were purchased from R\&D Systems, Inc. (Minneapolis, MN, USA), phosphorylated (p)-ERK (1:200; cat. no. 612358) was purchased from BD Biosciences (Franklin Lakes, NJ, USA) and total (t)-ERK (1:100; cat. no. 1051) was obtained from Boster Biological Technology (Pleasanton, CA, USA). The GAPDH antibody (1:200; cat. no. 2118) was purchased from Cell Signaling Technology, Inc. (Danvers, MA, USA). Antibodies against p-p38 (1:100; cat. no. AM063), t-p38 (1:100; cat. no. AM065), p-JNK (1:100; cat. no. AF1762) and t-JNK (1:100; cat. no. AF1048) were purchased from Beyotime Institute of Biotechnology (Haimen, China). Rabbit anti-mouse immunoglobulin G (IgG) secondary antibody [horseradish peroxidase (HRP)-conjugated; 1:10,000; cat. no. 58802) and mouse anti-rabbit IgG secondary antibody (HRP-conjugated; 1:10,000; cat. no. 5127) were obtained from Cell Signaling Technology, Inc. Rabbit anti-goat IgG secondary antibody (HRP-conjugated; 1:10,000; cat. no. HAF017) was purchased from Bio-Techne, Minneapolis, MN, USA.

Reverse transcription-quantitative polymerase chain reaction $(R T-q P C R)$. The sciatic nerves from experimental mice in each group were frozen in liquid nitrogen. Total mRNA was isolated with TRIzol ${ }^{\circledR}$ reagent (Invitrogen; Thermo Fisher Scientific, Inc., Waltham, MA, USA) and subsequently reverse-transcribed using the PrimeScript ${ }^{\mathrm{TM}}$ RT reagent kit (Takara Bio, Inc., Otsu, Japan) according to the manufacturer's protocol. qPCR was performed with SYBR Premix ExTaq (Takara Biotechnology Co., Ltd. Dalian, China) and the following primers provided by Sangon Biotech Co., Ltd. (Shanghai, China): IGF-1R, forward, 5'-ATCCTGTGTTCT TCTATGTCC-3' and reverse, 5'-CCAACCTGCTGTTATTTC TC-3'; and GAPDH forward, 5'-TCCTGCACCACCAACTGC TTAG-3' and reverse, 5'-AGTGGCAGTGATGGCATGGAC T-3'. The thermocycling conditions were: $95^{\circ} \mathrm{C}$ for $\geq 30 \mathrm{sec}$, 40 cycles at $95^{\circ} \mathrm{C}$ for $15 \mathrm{sec}$ and $60^{\circ} \mathrm{C}$ for $60 \mathrm{sec}$ in a DNA 
A

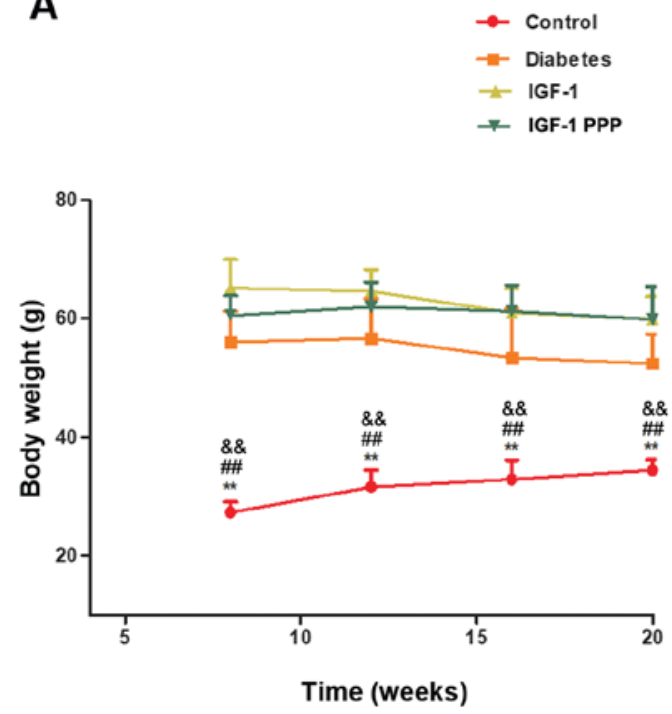

B

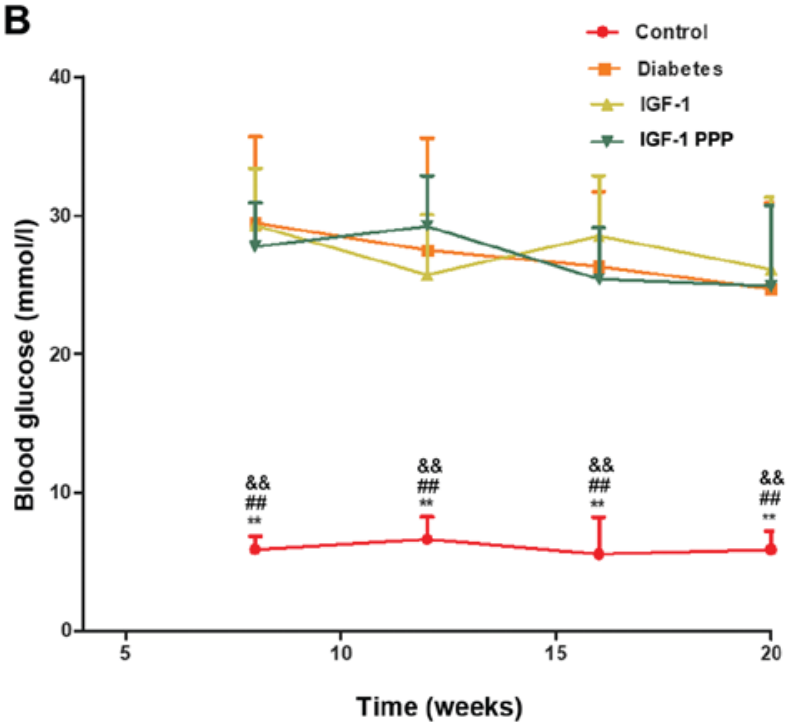

Figure 1. Body weight and blood glucose of mice are measured. Comparison of (A) body weight and (B) blood glucose in the four groups at $8,12,16$ and 20 weeks of age. The data are presented as the mean \pm standard deviation. ${ }^{* *} \mathrm{P}<0.01$, control group vs. diabetes group; ${ }^{\# \#} \mathrm{P}<0.01$, IGF-1 group vs. control group; ${ }^{\&} \mathrm{P}<0.01$, IGF-1-PPP group vs. control group. IGF-1, insulin-like growth factor 1; PPP, picropodophyllin.

iCycler apparatus (7900 HT Sequence Detection System, ABI, USA). Genes were quantified using the $2^{-\Delta \Delta} \mathrm{Cq}$ method (19) with GAPDH as the housekeeping gene. For each set of reactions, the samples were analyzed in triplicate.

Statistical analysis. SPSS software version 18.0 for Windows (SPSS, Inc., Chicago, IL, USA) was used for statistical analysis. Data are expressed as the mean \pm standard deviation and analyzed for statistical significance by one-way analysis of variance, followed by Tukey's post-hoc test. Experiments were repeated three times. $\mathrm{P}<0.05$ was considered to indicate a statistically significant difference.

\section{Results}

Body weight and blood glucose of mice. No significant differences were observed in body weight (Fig. 1A) and fasting blood glucose levels (FBG; Fig. 1B) between the diabetes, IGF-1 and IGF-1-PPP groups. Over time, the weight of the diabetes, IGF-1 and IGF-1-PPP groups decreased; however, were still significantly increased compared with the control at different time points $(\mathrm{P}<0.01)$. For the control group, although the weight of control group was increased, the differences were not significant at different time points $(\mathrm{P}>0.05)$. For the diabetes, IGF-1 and IGF-1-PPP groups, although these three groups exhibited weight loss, the differences were not significant at different time points $(\mathrm{P}>0.05)$.

Effects of IGF-1 and the IGF-1R antagonism PPP on behavior. Compared with the control and IGF-1 groups, the PWL values of the diabetes and IGF-1-PPP groups were markedly extended after 12 weeks of intervention. The maximum pain threshold was increased by $6.7 \pm 1.1 \mathrm{sec}$ $(n=8-10$; Fig. 2A) compared with the control group; the difference was statistically significant $(\mathrm{P}<0.05)$. Compared with the control and IGF-1 groups, the PWT of the diabetes and IGF-1-PPP groups significantly decreased after 12 weeks of intervention $(\mathrm{P}<0.05)$. The maximum pain threshold was decreased by $3.2 \pm 0.4 \mathrm{~g}(\mathrm{n}=8-10$; Fig. $2 \mathrm{~B})$ compared with the control group.

Effects of IGF-1 and IGF-1R antagonism on MNCV. No significant differences were observed in the rates of conduction in sciatic nerves between the four groups at 8 weeks of age prior to treatment $(\mathrm{P}>0.05)$. Following 12 weeks of treatment (20 weeks old), the sciatic MNCV in the diabetes, IGF-1 and IGF-1-PPP groups was significantly lower compared with the control group at 12 weeks of age $(\mathrm{P}<0.05)$. Additionally, following 12 weeks of treatment, the sciatic MNCV in the diabetes and IGF-1-PPP groups was significantly lower compared with the control group; however, the levels of the MNCV of the IGF-1 group significantly increased at 20 weeks of age and were similar to the control group ( $\mathrm{P}<0.01$; Fig. 3$)$.

Effects of IGF-1 and IGF-1R antagonism on sciatic nerve morphology. Following 12 weeks of treatment, the sciatic nerve axis of the control group was relatively complete and the myelin sheath exhibited regular formation at 20 weeks of age under a transmission electron microscope. The myelin sheath in the sciatic nerve of the diabetes group exhibited irregular vacuoles and separation of the intima from the myelin sheath. Following rIGF-1 intervention, however, the sciatic nerves of the diabetes group revealed signs of recovery, which manifested as an increase in Schwann cells indicated by the white arrows, an intact myelin sheath and notable reduced number of vacuoles. The recovery of impaired sciatic nerves was attenuated following the administration of the IGF-1R antagonist, PPP (Fig. 4).

Effects of IGF-1 and IGF-1R antagonism on IGF-1 and IGFBP-3 protein expression levels. The concentration of serum IGF-1 in the diabetes group at 20 weeks of age 
A

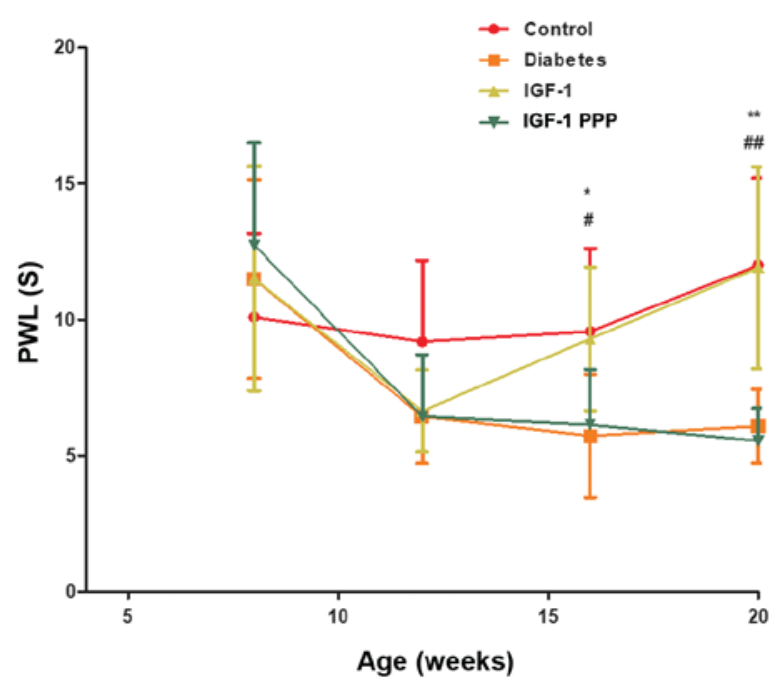

B

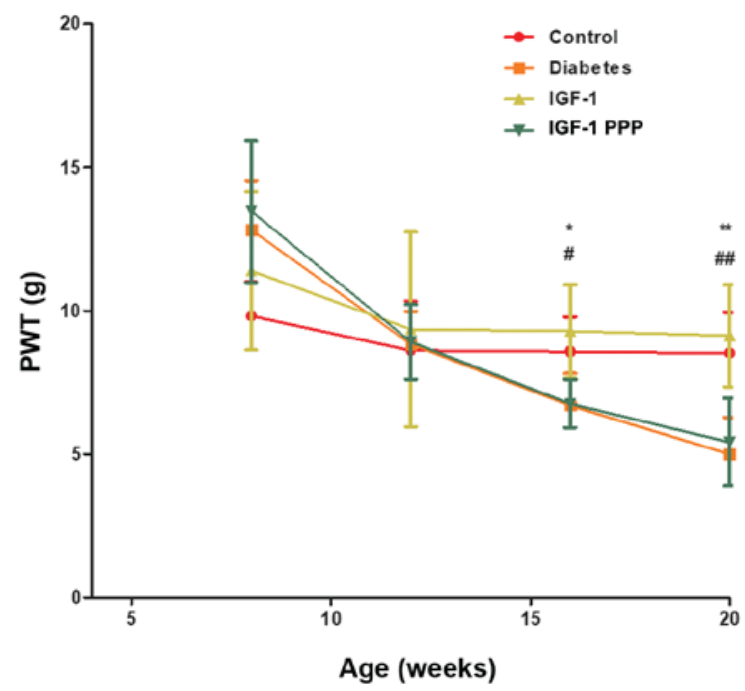

Figure 2. Effects of IGF-1 and the IGF-1R antagonism PPP on behavior. Comparison of (A) PWL and (B) PWT in the four groups at $8,12,16$ and 20 weeks of age. The data are presented as the mean \pm standard deviation. ${ }^{*} \mathrm{P}<0.05$ and ${ }^{* * *} \mathrm{P}<0.01$, control group vs. diabetes group. ${ }^{\#} \mathrm{P}<0.05$ and ${ }^{\# \#} \mathrm{P}<0.01$, IGF-1 group vs. diabetes group. IGF-1, insulin-like growth factor 1; PPP, picropodophyllin; PWL, paw withdrawal latency; PWT paw withdrawal threshold.

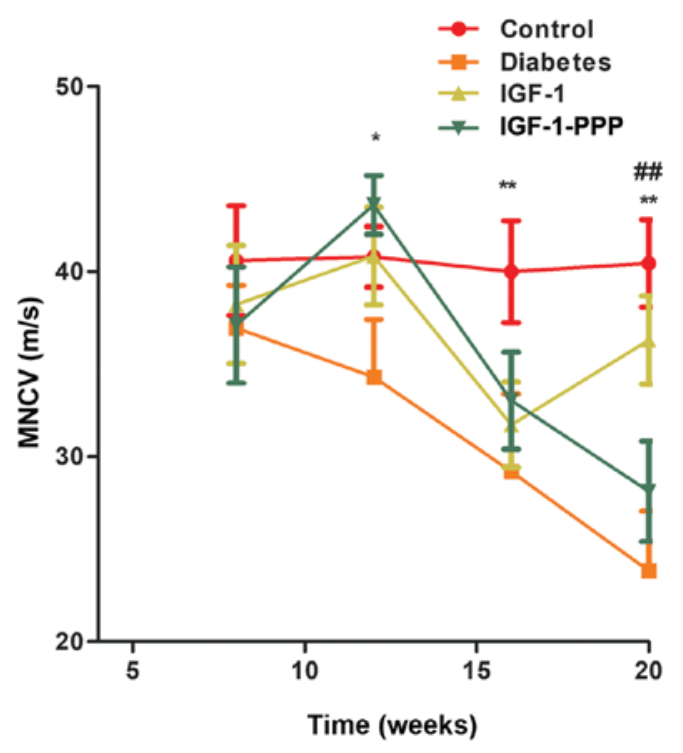

Figure 3. Conduction of sciatic nerves in the 4 groups at $8,12,16$ and 20 weeks of age. The data are presented as the mean \pm standard deviation. ${ }^{*} \mathrm{P}<0.05$ and ${ }^{* *} \mathrm{P}<0.01$, control group vs. diabetes group. ${ }^{\# \#} \mathrm{P}<0.01$, IGF-1 group vs. diabetes group. IGF-1, insulin-like growth factor 1 ; MNCV, motor nerve conduction velocity; PPP, picropodophyllin.

was significantly lower compared with the control group $(\mathrm{P}<0.05)$. Following 12 weeks of intervention with IGF-1, the concentration of serum IGF-1 in the IGF-1 group increased compared with the diabetes group, and the IGF-1-PPP group significantly decreased compared with the IGF-1 group (Fig. 5A).

In addition, the serum concentration of IGFBP-3 in the diabetes group at 20 weeks of age was significantly higher compared with the control group $(\mathrm{P}<0.05)$. Following 12 weeks of intervention with IGF-1, the serum concentration of IGFBP-3 in the IGF-1 group was decreased compared with the diabetes group, and decreased further in the IGF-1-PPP group compared with the IGF-1 group (Fig. 5B).

Effects of IGF-1 and IGF-1R antagonism on IGF-1R, JNK, $E R K$ and p38. No significant alterations were observed in the protein expression levels of t-JNK, t-ERK and t-p38 in the sciatic nerves of each group. ( $\mathrm{P}>0.05$; Fig. 6) The protein expression levels of $\mathrm{p}-\mathrm{JNK}$ and $\mathrm{p}$-ERK in the sciatic nerves of the diabetes group were significantly lower compared with the control group. The difference was statistically significant $(\mathrm{P}<0.01)$. Compared with the diabetes group, the expression levels of p-JNK and p-ERK in the IGF-1 group were significantly increased $(\mathrm{P}<0.01)$. Compared with the IGF-1 group, the expression levels of p-JNK and p-ERK in the IGF-1-PPP group were significantly decreased $(\mathrm{P}<0.01$; Fig. 6$)$.

The phosphorylation levels of p38 were relatively high in the control group compared with the other three groups; however, the difference was not statistically significant. No significant differences were observed between the other three groups (Fig. 6).

The expression levels of IGF-1R protein in the sciatic nerves of the diabetes group were significantly lower compared with the control group $(\mathrm{P}<0.01)$. Additionally, compared with the diabetes group, the IGF-1R protein expression levels in IGF-1 group were significantly increased $(\mathrm{P}<0.05)$. Conversely, the IGF-1R protein expression levels in the IGF-1-PPP group were decreased compared with the IGF-1 group; the difference was not statistically significant ( $\mathrm{P}>0.05$; Fig. 6).

Effects of IGF-1 and IGF-1R antagonism on IGF-1R $m R N A$ expression. The mRNA expression levels of IGF-1R were observed to be significantly decreased in the sciatic nerve of the diabetes group compared with the control (Fig. 7). Conversely, a significant increase in the expression levels of IGF-1R in the sciatic nerve of the IGF-1 group was detected compared with the diabetes group (Fig. 7). Treatment with PPP induced a significant decrease in the 


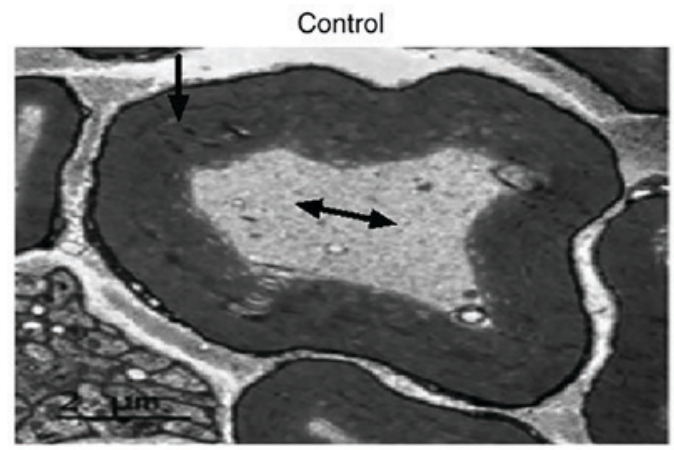

IGF-1

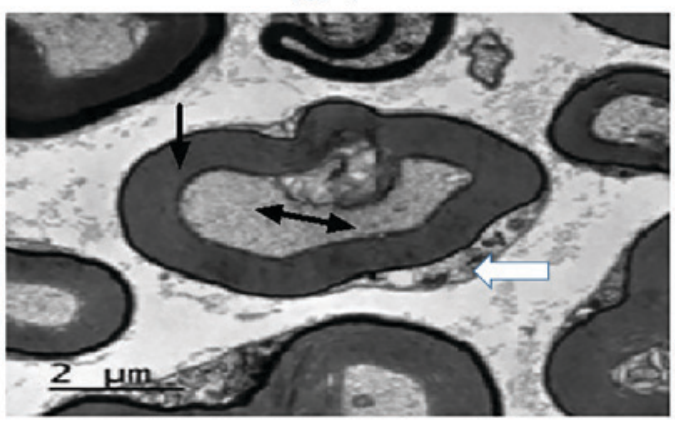

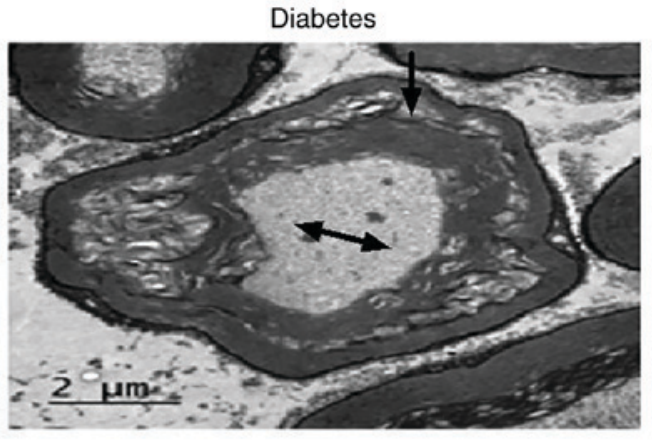

IGF-1-PPP

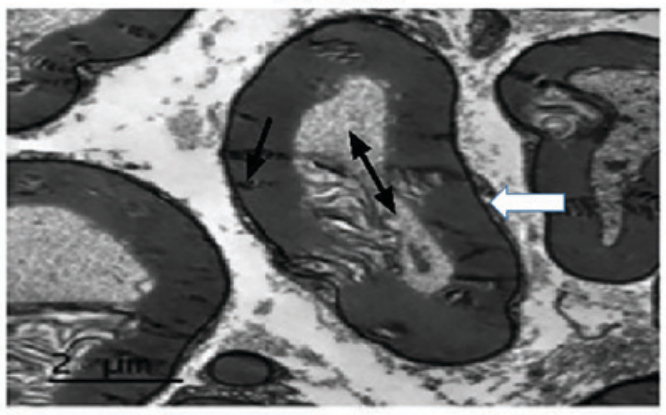

Figure 4. Effects of IGF-1 and IGF-1 receptor antagonism on sciatic nerve morphology at 20 weeks of age. Single arrows indicate the myelin sheath in the sciatic nerve. Double-headed arrows indicate the axon, white arrows indicate Schwann cells. Scale bar, $2 \mu \mathrm{m}$. IGF-1, insulin-like growth factor 1; PPP, picropodophyllin.

A

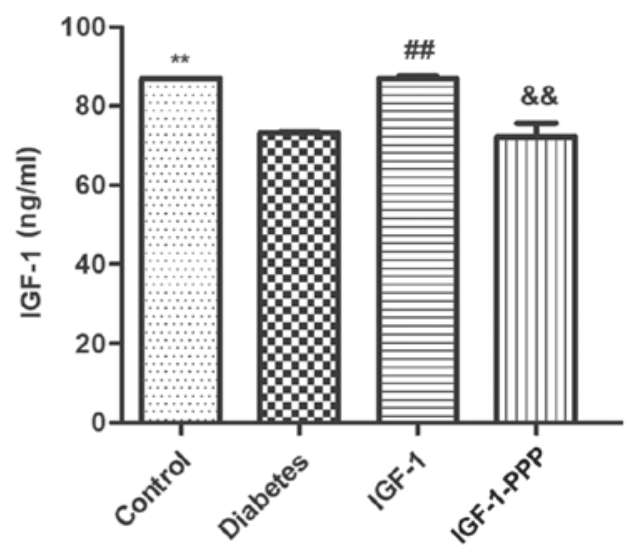

B

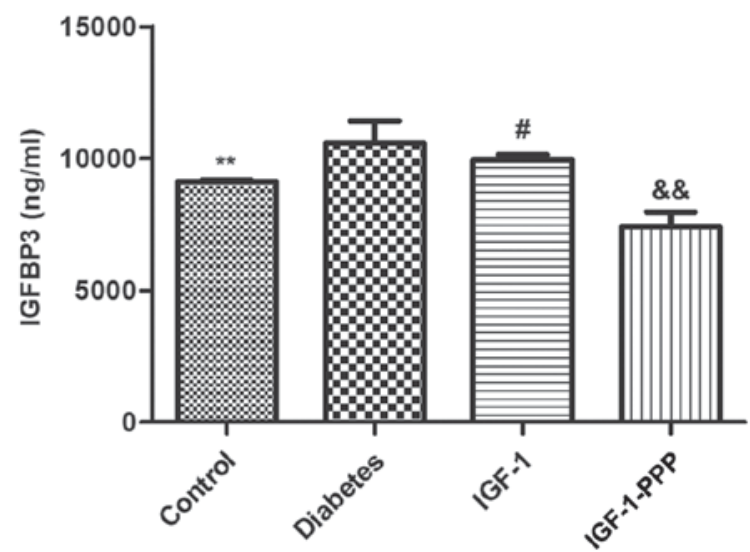

Figure 5. Effects of IGF-1 and IGF-1R antagonism on IGF-1 and IGFBP-3 protein levels. (A) IGF-1 and (B) IGFBP-3 protein expression levels in the four groups at 20 weeks of age. The data are presented as the mean \pm standard deviation. ${ }^{* *} \mathrm{P}<0.01$, control group vs. diabetes group; ${ }^{*} \mathrm{P}<0.05$ and ${ }^{\sharp \# \#} \mathrm{P}<0.01$, IGF-1 group vs. diabetes group; ${ }^{\& \&} \mathrm{P}<0.01$, IGF-1-PPP group vs. IGF-1 group. IGF-1, insulin-like growth factor 1; IGFBP-3, IGF binding protein 3; PPP, picropodophyllin.

expression levels of IGF-1R compared with the IGF-1 group (Fig. 7).

\section{Discussion}

DPN is one of the principal chronic complications associated with diabetes, with high morbidity rates among patients with diabetes. DPN reduces quality of life due to pain, sensory loss, gait instability, fall-associated injuries, foot ulceration and amputation, and the causative mechanisms of DPN remain unclear $(20,21)$. To the best of our knowledge, there is currently no effective therapy for the treatment of DPN. IGF-1 has been reported to be closely associated with
DPN (14). A previous study evaluated the association of three diabetic complications on the serum free IGF-I and IGFBP-3 expression levels in patients with type 1 diabetes (22). It was demonstrated that patients with type 1 diabetes mellitus exhibited lower serum free IGF and IGFBP-3 expression compared with healthy individuals (22). Neuropathy was reported to be associated with a significant reduction in serum free IGF-1 (22). It has been proposed that in a streptozotocin (STZ)-treated mouse model of type 1 diabetes mellitus, mice (C57BL/6 N) developed hypoalgesia, which was associated with the decline in serum IGF-1 expression levels (23). The sensory complications of DPN in the mice model of type 1 diabetes mellitus may be corrected 
A

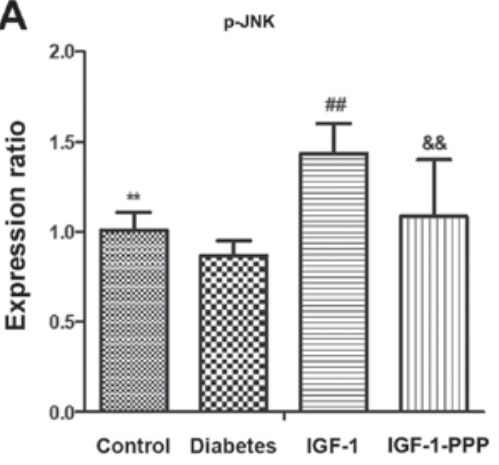

C

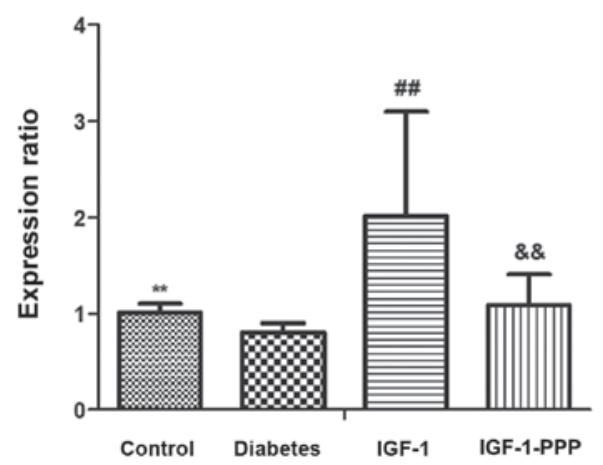

B
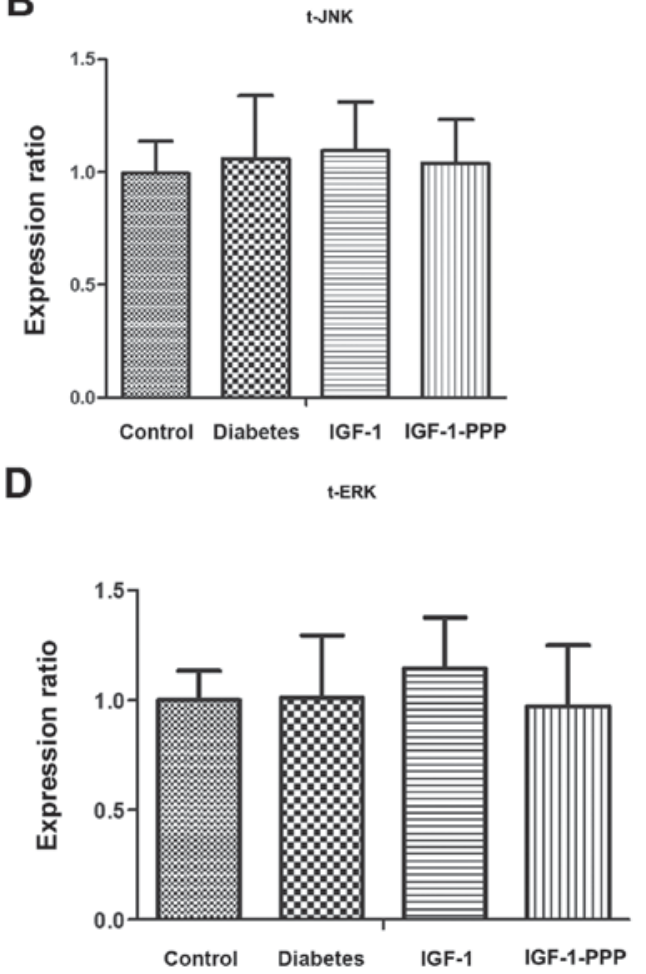

E

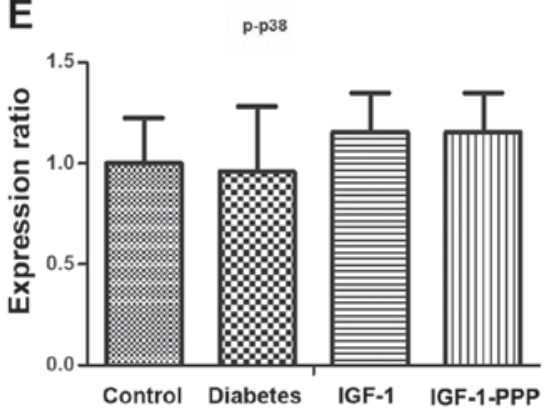

G

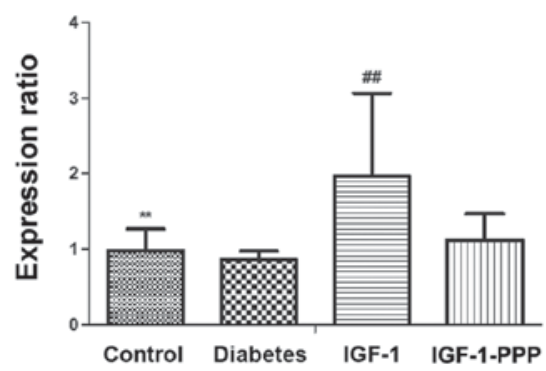

F

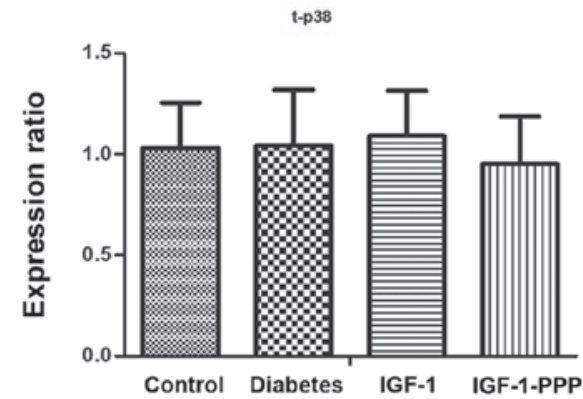

H

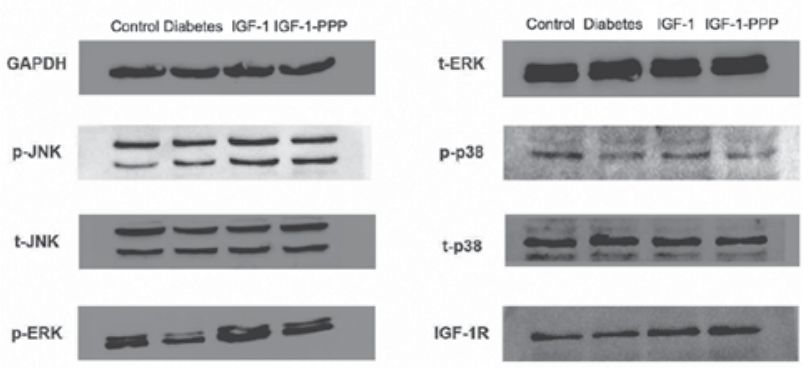

Figure 6. Comparison of JNK, ERK and p38 expression and phosphorylation, and IGF-1R expression. Protein expression levels of (A and B) JNK, (C and D) ERK, (E and F) p38 and (G) IGF-1R in sciatic nerves of the 4 groups at 20 weeks of age were detected by western blot analysis and (H) semi-quantification was performed. The data are presented as the mean \pm standard deviation. ${ }^{* *} \mathrm{P}<0.01$, control group vs. diabetes group; ${ }^{\# \#} \mathrm{P}<0.01$, IGF-1 group vs. diabetes group; \&\&P<0.01, IGF-1-PPP group vs. IGF-1 group. p, phosphorylated; t, total; JNK, c-Jun N-terminal kinase; ERK, extracellular signal-regulated kinase; IGF-1R, insulin-like growth factor 1 receptor; PPP, picropodophyllin.

by restoring circulating IGF-1 expression levels to normal via a tail-vein injection of a recombinant adeno-associated virus, AAV8-smIGF-1 (23). The partial reversal of established DPN suggested a role for IGF-1 in the pathogenesis and therapy of this condition. Investigation into the role of IGF-1 may aid the development of clinical treatments for
DPN (24). Furthermore, an STZ-treated mouse (ICR mouse) model of DPN demonstrated that an intrathecal injection of a recombinant adeno-associated virus, rAAV2/1, regulated the expression of IGF-1 via the protein kinase B (Akt)/phosphatidylinositol 3-kinase (PI3K) signaling pathway to promote the expression of vascular endothelial growth factor and the 
regeneration of nerves, thus improving motor and sensory neuropathy (25).

The aim of the present study was to evaluate the protective effects of IGF-1 in alleviating the peripheral neuropathy symptoms typically associated with diabetes. For this purpose, a mouse model of type 2 diabetic neuropathy was established and behavioral analysis was performed in the present study. Existing literature regarding the effects of experimentally-induced diabetes on pain thresholds in rodent models remains controversial (26). In the present study, alterations in algesia were determined as PWL and PWT. A previous study demonstrated that the mice developed hyperalgesia at 10 weeks of age (26). However, in the present study, the mice developed hyperalgesia until 16 weeks of age. PWL and PWT are more suitable for measuring the pain threshold of acute pain, and the duration of diabetes of 16-20 weeks in the mice is a sufficient time to produce chronic pain, different from the previous short-term mice study (26). Furthermore, decreases in MNCV in the diabetes group were observed in the present study. Therefore, abnormal electrophysiologic parameters, particularly those of motor nerves, may be considered as an early indicator of DPN. In addition, typical structural abnormalities were observed, including demyelination and vacuolar alterations; however, following treatment with IGF-1, the sciatic nerves exhibited indications of recovery. This may due to the expression of IGF-1R on Schwann cells; activation of this receptor has been revealed to promote myelination (27). Furthermore, as one of the growth factors expressed by Schwann cells, IGF-1 is reported to be involved in the metabolism of neuronal myelin, suggesting that IGF-1 may be involved in the onset and development of DPN (28).

Additionally, it was reported that reduced serum IGF-1 was associated with alterations in electrophysiologic and structural parameters in the diabetes group. Therefore, the present study investigated the effects of IGF-1 on DPN following an intraperitoneal injection of IGF-1. Following treatment, the serum concentration IGF-1 in the IGF-1 group was increased, which was consistent with the observed improvements of sciatic nerve morphology.

The results of the present study suggested that treatment with IGF-1 may benefit DPN-induced $\mathrm{db} / \mathrm{db}$ mice. In addition, treatment with IGF-1 following the onset of diabetes may reverse neuronal deficits, as observed in the present study. This was consistent with an earlier observation in a rat model, in which IGF replacement therapy was proposed to reverse or prevent diabetic neuropathy, independent of hyperglycemia or weight loss (29). The present study additionally demonstrated that IGF-1 may be a potential therapeutic agent for diabetic neuropathy; and similarly, a previous study reported that rIGF-1 may restore sensory and motor NCVs in IGF-I deficient mice (30). These results suggested that the effects of IGF-1 as a treatment for DPN may occur via the activation of IGF-1R.

IGF-1 targets the extracellular binding site of IGF-1R and activates the downstream intracellular protein insulin receptor substrate (IRS-1) via phosphorylation of the D transmembrane subunit of IGF-1R (31). IRS-1 transduces the signal of IGF-1 via two downstream cascades, the Akt/PI3K and mitogen-activated protein kinase (MAPK) signaling pathways (31). The association between the Akt and MAPK signaling pathways has been reported, and their equilibrium is important for maintaining

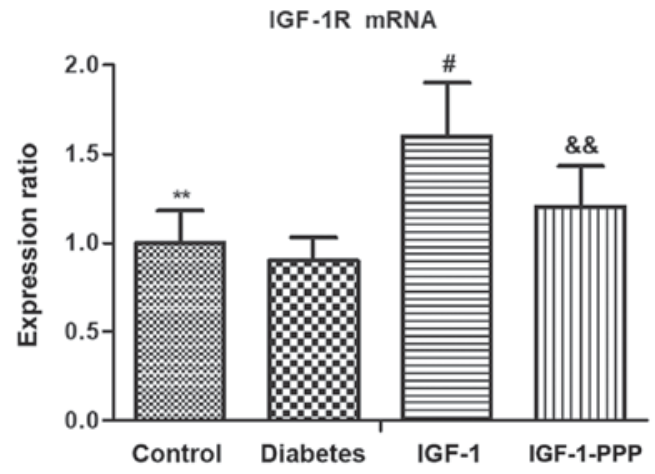

Figure 7. IGF-1R mRNA expression in the 4 groups at 20 weeks of age. The data are presented as the mean \pm standard deviation. ${ }^{* *} \mathrm{P}<0.01$, control group vs. diabetes group; ${ }^{\#} \mathrm{P}<0.05$, IGF-1 group vs. diabetes group; ${ }^{\&} \mathrm{P}<0.01$, IGF-1-PPP group vs. IGF-1 group. IGF-1, insulin-like growth factor 1; IGF-1R, insulin-like growth factor 1 receptor; PPP, picropodophyllin.

the physiological function of normal cells (32). Previous studies have demonstrated that IGF-1 signaling is mostly dependent on the Akt/PI3K pathway, to serve a pro-apoptotic role in neurons $(33,34)$; however, further investigation into the association between the MAPK signaling pathway and IGF-1 is required. The MAPK family of proteins constitutes a set of serine and threonine protein kinases with several subfamilies. Among these proteins are three important members, which maintain the normal physiological function of cells, ERK, JNK and p38 (35). Therefore, it is important to investigate the role of MAPK signaling in DPN. To gain insight into the respective roles served by the MAPK signaling pathway, via western blotting, the present study revealed that the role of IGF-1, which offers maintenance support for the sciatic nerve (36), may be associated with increased expression levels of p-JNK and p-ERK, not p-p38. As only preliminary signaling studies on the sciatic nerve of animals have been conducted, further investigations of ion channels in vitro is required to understand the protective effect of IGF-1 against neuropathy in diabetic mice and its potential underlying mechanisms.

In conclusion, the present results suggested that, in a diabetic animal model, IGF-1 may be a highly effective therapeutic modality for treating diabetic peripheral neuropathy. The effects of IGF-1, which may provide trophic support for the sciatic nerve, may be associated with the increased phosphorylation levels of JNK and ERK, not p38. These neurotrophic effects of IGF-1 may be attenuated following the administration of an IGF-1R antagonist. Although at present, systemic administration of free IGF-1 in patients has restricted therapeutic potential due to its instability in during circulation and side effects, in the future, IGF-1, provided by either an agonist or by agents that may serve to upregulate IGF-1, may provide therapeutic benefits in DPN.

\section{Acknowledgements}

Not applicable.

\section{Funding}

The present study was supported by the Shanghai Nature Science Fund projects (grant no. 14ZR1434000; Shanghai, 
China) and the Fund of Science and Technology Department of Pudong New Area (grant no. PKJ2015-Y19; Shanghai, China).

\section{Availability of data and materials}

The datasets used and/or analyzed during the current study are available from the corresponding author on reasonable request.

\section{Authors' contributions}

HW conceived and designed the experiments. ZT, FC and JL performed the experiments. $\mathrm{HW}, \mathrm{ZT}$ and $\mathrm{HZ}$ analyzed the data. JT and HL acquired the data. YZ and BF interpreted the data. All authors read and approved the final version of the manuscript.

\section{Ethics approval and consent to participate}

All experimental procedures were approved by the Ethics of Animal Experiments Committee of the Tongji University School of Medicine. The present study was conducted according to internationally recognized guidelines on animal welfare, in addition to the regulations regarding animal welfare in Shanghai, and the Guidelines of the Chinese Council on Animal Care.

\section{Patient consent to participate}

Not applicable.

\section{Competing interests}

The authors declare that they have no competing interests.

\section{References}

1. Xu Y, Wang L, He J, Bi Y, Li M, Wang T, Wang L, Jiang Y, Dai M, Lu J, et al: Prevalence and control of diabetes in Chinese adults. JAMA 310: 948-959, 2013.

2. Galuppo M, Giacoppo S, Bramanti P and Mazzon E: Use of natural compounds in the management of diabetic peripheral neuropathy. Molecules 19: 2877-2895, 2014.

3. Bongaerts BW, Rathmann W, Kowall B, Herder C, Stöckl D, Meisinger $\mathrm{C}$ and Ziegler D: Postchallenge hyperglycemia is positively associated with diabetic polyneuropathy: The KORA F4 study. Diabetes Care 35: 1891-1893, 2012.

4. Duby JJ, Campbell RK, Setter SM, White JR and Rasmussen KA: Diabetic neuropathy: An intensive review. Am J Health Syst Pharm 61: 160-173; quiz 175-176, 2004.

5. Morrison JF, Shehab S, Sheen R, Dhanasekaran S, Shaffiullah M and Mensah-Brown E: Sensory and autonomic nerve changes in the monosodium glutamate-treated rat: A model of type II diabetes. Exp Physiol 93: 213-222, 2008.

6. Beiswenger KK, Calcutt NA and Mizisin AP: Epidermal nerve fiber quantification in the assessment of diabetic neuropathy. Acta Histochem 110: 351-362, 2008.

7. Grisold A, Callaghan BC and Feldman EL: Mediators of diabetic neuropathy: Is hyperglycemia the only culprit? Curr Opin Endocrinol Diabetes Obes 24: 103-111, 2017.

8. Román-Pintos LM, Villegas-Rivera G, Rodríguez-Carrizalez AD, Miranda-Díaz AG and Cardona-Muñoz EG: Diabetic polyneuropathy in Type 2 diabetes mellitus: Inflammation, oxidative stress and mitochondrial function. J Diabetes Res 2016: 3425617, 2016.

9. Chung SS, Ho EC, Lam KS and Chung SK: Contribution of polyol pathway to diabetes-induced oxidative stress. J Am Soc Nephrol 14 (8 Suppl 3): S233-S236, 2003.
10. Yakar S, Pennisi P, Wu Y, Zhao $\mathrm{H}$ and LeRoith $\mathrm{D}$ : Clinical relevance of systemic and local IGF-I. Endocr Dev 9: 11-16, 2005.

11. Hao CN, Geng YJ, Li F, Yang T, Su DF, Duan JL and Li Y: Insulin-like growth factor-1 receptor activation prevents hydrogen peroxide-induced oxidative stress, mitochondrial dysfunction and apoptosis. Apoptosis 16: 1118-1127, 2011.

12. Puche JE, García-Fernández M, Muntané J, Rioja J, González-Barón S and Castilla Cortazar I: Low doses of insulin-like growth factor-I induce mitochondrial protection in aging rats. Endocrinology 149: 2620-2627, 2008.

13. Carro E, Trejo JL, Núñez A and Torres-Aleman I: Brain repair and neuroprotection by serum insulin-like growth factor I. Mol Neurobiol 27: 153-162, 2003.

14. Ishii DN: Implication of insulin-like growth factors in the pathogenesis of diabetic neuropathy. Brain Res Brain Res Rev 20: 47-67,1995.

15. Papanas $\mathrm{N}$ and Ziegler D: Risk factors and comorbidities in diabetic neuropathy: An update 2015. Rev Diabet Stud 12: 48-62, 2015.

16. Menu E, Jernberg-Wiklund H, Stromberg T, De Raeve H, Girnita L, Larsson O, Axelson M, Asosingh K, Nilsson K, Van Camp B and Vanderkerken K: Inhibiting the IGF-1 receptor tyrosine kinase with the cyclolignan PPP: An in vitro and in vivo study in the 5T33MM mouse model. Blood 107: 655-660, 2006.

17. Hargreaves K, Dubner R, Brown F, Flores C and Joris J: A new and sensitive method for measuring thermal nociception in cutaneous hyperalgesia. Pain 32: 77-88, 1988.

18. Kanazawa Y, Takahashi-Fujigasaki J, Ishizawa S, Takabayashi N, Ishibashi K, Matoba K, Kawanami D, Yokota T, Tajima N and Utsunomiya K: The Rho-kinase inhibitor fasudil restores normal motor nerve conduction velocity in diabetic rats by assuring the proper localization of adhesion-related molecules in myelinating Schwann cells. Exp Neurol 247: 438-446, 2013.

19. Livak KJ and Schmittgen TD: Analysis of relative gene expression data using real-time quantitative PCR and the 2(-Delta Delta C(T)) method. Methods 25: 402-408, 2001.

20. Tesfaye S, Boulton AJ, Dyck PJ, Freeman R, Horowitz M, Kempler P, Lauria G, Malik RA, Spallone V, Vinik A, et al: Diabetic neuropathies: Update on definitions, diagnostic criteria, estimation of severity and treatments. Diabetes Care 33: 2285-2293, 2010.

21. Rathur HM and Boulton AJ: The neuropathic diabetic foot. Nat Clin Pract Endocrinol Metab 3: 14-25, 2007.

22. Capoluongo E, Pitocco D, Santonocito C, Concolino P, Santini SA, Manto A, Lulli P, Ghirlanda G, Zuppi C and Ameglio F: Association between serum free IGF-I and IGFBP-3 levels in type-I diabetes patients affected with associated autoimmune diseases or diabetic complications. Eur Cytokine Netw 17: $167-174,2006$.

23. Chu Q, Moreland R, Yew NS, Foley J, Ziegler R and Scheule RK: Systemic insulin-like growth factor-1 reverses hypoalgesia and improves mobility in a mouse model of diabetic peripheral neuropathy. Mol Ther 16: 1400-1481, 2008.

24. Schmidt RE, Dorsey DA, Beaudet LN, Plurad SB, Parvin CA and Miller MS: Insulin-like growth factor I reverses experimental diabetic autonomic neuropathy. Am J Pathol 155: 1651-1660, 1999.

25. Homs J, Pagès G, Ariza L, Casas C, Chillón M, Navarro X and Bosch A: Intrathecal administration of IGF-I by AAVrh10 improves sensory and motor deficits in a mouse model of diabetic neuropathy. Mol Ther Methods Clin Dev 1: 7, 2014.

26. O'Brien PD, Sakowski SA and Feldman EL: Mouse models of diabetic neuropathy. ILAR J 54: 259-272, 2014.

27. Russell JW, Cheng HL and Golovoy D: Insulin-like growth factor-I promotes myelination of peripheral sensory axons. J Neuropathol Exp Neurol 59: 575-584, 2000.

28. Delaney CL, Russell JW, Cheng HL and Feldman EL: Insulin-like growth factor-I and over-expression of Bcl-xL prevent glucose-mediated apoptosis in Schwann cells. J Neuropathol Exp Neurol 60: 147-160, 2001.

29. Zhuang HX, Snyder CK, Pu SF and Ishii DN: Insulin-like growth factors reverse or arrest diabetic neuropathy: Effects on hyperalgesia and impaired nerve regeneration in rats. Exp Neurol 140: 198-205, 1996.

30. Gao WQ, Shinsky N, Ingle G, Beck K, Elias KA and Powell-Braxton L: IGF-I deficient mice show reduced peripheral nerve conduction velocities and decreased axonal diameters and respond to exogenous IGF-I treatment. J Neurobiol 39: 142-152, 1999. 
31. Rabiee A, Krüger M, Ardenkjær-Larsen J, Kahn CR and Emanuelli B: Distinct signalling properties of insulin receptor substrate (IRS)-1 and IRS-2 in mediating insulin/IGF-1 action. Cell Signal 47: 1-15, 2018.

32. Zhou YL, Liu SQ, Yuan B and Lu N: The expression of insulin-like growth factor-1 in senior patients with diabetes and dementia. Exp Ther Med 13: 103-106, 2017.

33. Pfäffle R, Kiess W and Klammt J: Downstream insulin-like growth factor. Endocr Dev 23: 42-51, 2012.
34. Leinninger GM, Backus C, Uhler MD, Lentz SI and Feldman EL: Phosphatidylinositol 3-kinase and Akt effectors mediate insulin-like growth factor-I neuroprotection in dorsal root ganglia neurons. FASEB J 18: 1544-1546, 2004.

35. Qi M and Elion EA: MAP kinase pathways. J Cell Sci 118 3569-3572, 2005.

36. Mohammadi R and Saadati A: Influence of insulin-like growth factor I on nerve regeneration using allografts: A sciatic nervemodel. J Craniofac Surg 25: 1510-1515, 2014. 